\title{
ASCO 2021: Highlights in central nervous system tumors
}

\author{
Maximilian J. Mair · Anna S. Berghoff
}

Received: 9 July 2021 / Accepted: 31 August 2021 / Published online: 11 October 2021

(C) The Author(s) 2021

\begin{abstract}
Summary More than 140 abstracts were presented in the Central Nervous System Tumors track during the 2021 American Society of Clinical Oncology (ASCO) virtual meeting. Here, we review our personal highlights of the presented data. In rare entities such as papillary craniopharyngioma and neurotrophic tyrocine receptor kinase (NTRK)-fusion-positive tumors, promising data on targeted therapies were reported. In addition, early data on olaparib in highgrade glioma and combinational immunotherapy approaches will be briefly reviewed. Furthermore, the eagerly awaited results of the EORTC-1709 phase III trial on the pan-proteasome inhibitor marizomib in newly diagnosed glioblastoma were shown at the meeting. Although no practice-changing trials were presented for glioma patients, new treatments are on the horizon and results from modern platform trials are awaited in the near future.
\end{abstract}

Keywords CNS tumors · Glioma - Glioblastoma . Targeted therapy $\cdot$ Immunotherapy

$\begin{array}{ll}\text { Abbreviations } \\ \text { 2-HG } & \text { 2-hydroxyglutarate } \\ \text { ASCO } & \text { American Society of Clinical Oncology } \\ \text { CDK } & \text { Cyclin dependent kinase } \\ \text { CNS } & \text { Central nervous system } \\ \text { DNA-PK } & \text { DNA-dependent protein kinase } \\ \text { EORTC } & \begin{array}{l}\text { European Organization for Research and } \\ \end{array} \\ & \text { Treatment of Cancer }\end{array}$

M. J. Mair, MD · A. S. Berghoff, MD, $\mathrm{PhD}(\bowtie)$

Department of Medicine I, Division of Oncology,

Medical University of Vienna, Waehringer Guertel

18-20, 1090 Vienna, Austria

anna.berghoff@meduniwien.ac.at

M. J. Mair, MD

maximilian.mair@meduniwien.ac.at
ERK
GBM
IDH
MEK
MTOR
NTRK
OS
PARP
PD-(L) 1
PFS
TMZ
VEGF

\section{BRAF/MEK inhibition in newly diagnosed} papillary craniopharyngioma

Craniopharyngiomas are rare tumors occurring at an incidence of $\sim 0.2 / 100,000$ person/years. Previously, the BRAF V600E mutation could be detected in $95 \%$ of papillary craniopharyngiomas [1]. This genetic alteration has been characterized in a variety of solid tumors, and specific inhibitors such as vemurafenib and encorafenib have reached approval status. Neurosurgical resection is the primary treatment option for craniopharyngiomas; however in rare cases complete surgery is not possible. In the Alliance A071601 phase II trial, 16 patients with progressive papillary craniopharyngioma without previous radiotherapy were treated with combined BRAF/MEK inhibition (vemurafenib/cobimetinib) in a 28-day cycle (Table 1) [2]. All individuals who underwent more than one cycle of treatment showed objective responses at a median volumetric tumor reduction of $83 \%$. Possibly treatment-related grade 3 adverse events were observed in 12/16 patients. Further data in previously irradiated patients is pending. Nevertheless, the data suggest that BRAF/MEK inhibition may be a feasible 
approach in these rare tumors if no local therapies are available.

\section{Larotrectinib in NTRK-fusion-positive primary central nervous system tumors}

Although rare, oncogenic fusions of the neurotrophic receptor tyrosine kinase gene (NTRK) with other genes occur across many solid tumor entities. Specific NTRK inhibitors such as larotrectinib are approved for use in children and adults with NTRK-fusion-positive tumors. Efficacy and safety data of NTRK inhibition have been recently reported, with objective responses in $79 \%$ of patients with extracranial tumors at a favorable safety profile [3]. Respective data in CNS tumors were presented at ASCO 2021 [4]. Data of 33 patients with NTRK-fusion-positive CNS tumors have been shown, of whom 26 patients $(78.8 \%)$ were pediatric. Most patients were diagnosed with high-grade gliomas (19/33, 57.6\%), followed by low-grade gliomas $(8 / 33,24.2 \%)$ and singular cases with glioneuronal and neuroepithelial tumors as well as other rare entities. Observed outcomes were complete responses in $3 / 33(9.1 \%)$ patients, partial responses in 7 (21.2\%) patients, stable disease in $20(60.6 \%)$ patients and progressive disease in $3(9.1 \%)$ patients. All complete responses were seen in pediatric patients. Notably, 23/28 (82.1\%) patients with measurable disease showed a decrease in tumor size, and median time to response was 1.9 months. Treatment-related grade 3-4 adverse events were seen in 3 patients, and no treatment discontinuations due to toxicity were reported. In conclusion, the presented data showed that larotrectinib is feasible and safe in the specific (but small) subgroup of NTRK-fusion-positive CNS tumors.

\section{PARP inhibition in IDH-mutant high-grade glioma (OLAGLI trial)}

Mutations in the isocitrate dehydrogenase (IDH) $1 / 2$ gene occur in about $30 \%$ of diffuse gliomas. Preclinical data suggested that poly-ADP-ribose polymerase (PARP) inhibition reduces tumor growth via suppression of the homologous recombination DNA repair pathway [5]. Based on these observations, a phase II trial in recurrent high-grade IDH-mutant gliomas after radiotherapy and at least one line of alkylating chemotherapy was performed [6]. Patients were treated with olaparib $300 \mathrm{mg}$ twice daily which was generally well tolerated. Still, efficacy signals were weak. Median progression-free survival was 2.3 months, while median overall survival reached 15.9 months with is comparable to historical controls in IDH-mutant glioblastoma (GBM) [7]. At a median follow-up of 11 months, olaparib treatment was discontinued in $30 / 35(85.7 \%)$ patients due to tumor progression, suggesting limited antitumoral activity.

\section{Combinational approaches in glioma immunotherapy}

So far, immunotherapy has failed to show clinically meaningful efficacy in glioma. In recurrent GBM, the CheckMate-143 trial comparing nivolumab with bevacizumab failed to reach its primary endpoint, although some durable responses could be observed [8], and similar results could be observed in newly diagnosed GBM [9, 10]. Therefore, current research focuses on improving patient selection and combinational approaches. Indeed, vascular endothelial growth factor (VEGF) has been shown to impact antitumoral immunity [11], providing the rationale for combining anti-VEGF treatment with immune checkpoint inhibitors. At ASCO 2021, the results of one combination trial of nivolumab with standard$(10 \mathrm{mg} / \mathrm{kg})$ and low-dose $(3 \mathrm{mg} / \mathrm{kg})$ bevacizumab were reported [12]. However, overall survival of both arms was comparable to historical controls of bevacizumab monotherapy. Interestingly, patients aged $>60$ had better overall survival with standard-dose as compared to low-dose bevacizumab, suggesting that age may influence on the efficacy of anti-VEGF/anti-PD-1 combination therapies in brain tumors.

Another phase I study analyzed the impact of the IDH inhibitors ivosidenib and vorasidenib on epigenetic, transcriptomic and tissue immune markers in IDH-mutated glioma [13]. The extent of IDH inhibition was determined by the levels of the oncometabolite 2-hydroxyglutarate $(2-\mathrm{HG})$ in tumor tissue. In patients with optimal 2-HG suppression, CD3+/CD8+ tumor-infiltrating lymphocytes were significantly increased as compared to samples with suboptimal 2-HG suppression. IDH inhibition was associated with upregulated expression of genes involved in antigen presentation and type I interferon signaling, supporting the design of future combination trials of immunotherapy with IDH inhibitors.

\section{Marizomib in newly diagnosed glioblastoma (EORTC-1709/CCTG CE.8)}

In newly diagnosed GBM, the postoperative standard of care still consists in combined radiochemotherapy [14]. In general, radiotherapy is applied at a dose of $60 \mathrm{~Gy}$ in 30 fractions with daily concomitant temozolomide (TMZ) chemotherapy, followed by six 28day cycles of adjuvant TMZ on days 1-5. Despite optimal treatment, median overall survival (OS) is still limited to 15 months, underlining the urgent need for innovative therapies. In a previous extended phase I trial [15], the addition of the brain-penetrating, irreversible pan-proteasome inhibitor marizomib to standard radiochemotherapy was assessed in newly diagnosed GBM. Based on favorable efficacy and safety, the EORTC-1709/CCTG CE.8 phase III trial presented at ASCO 2021 was aimed to substantiate the clinical efficacy of marizomib added to TMZ-based ra- 


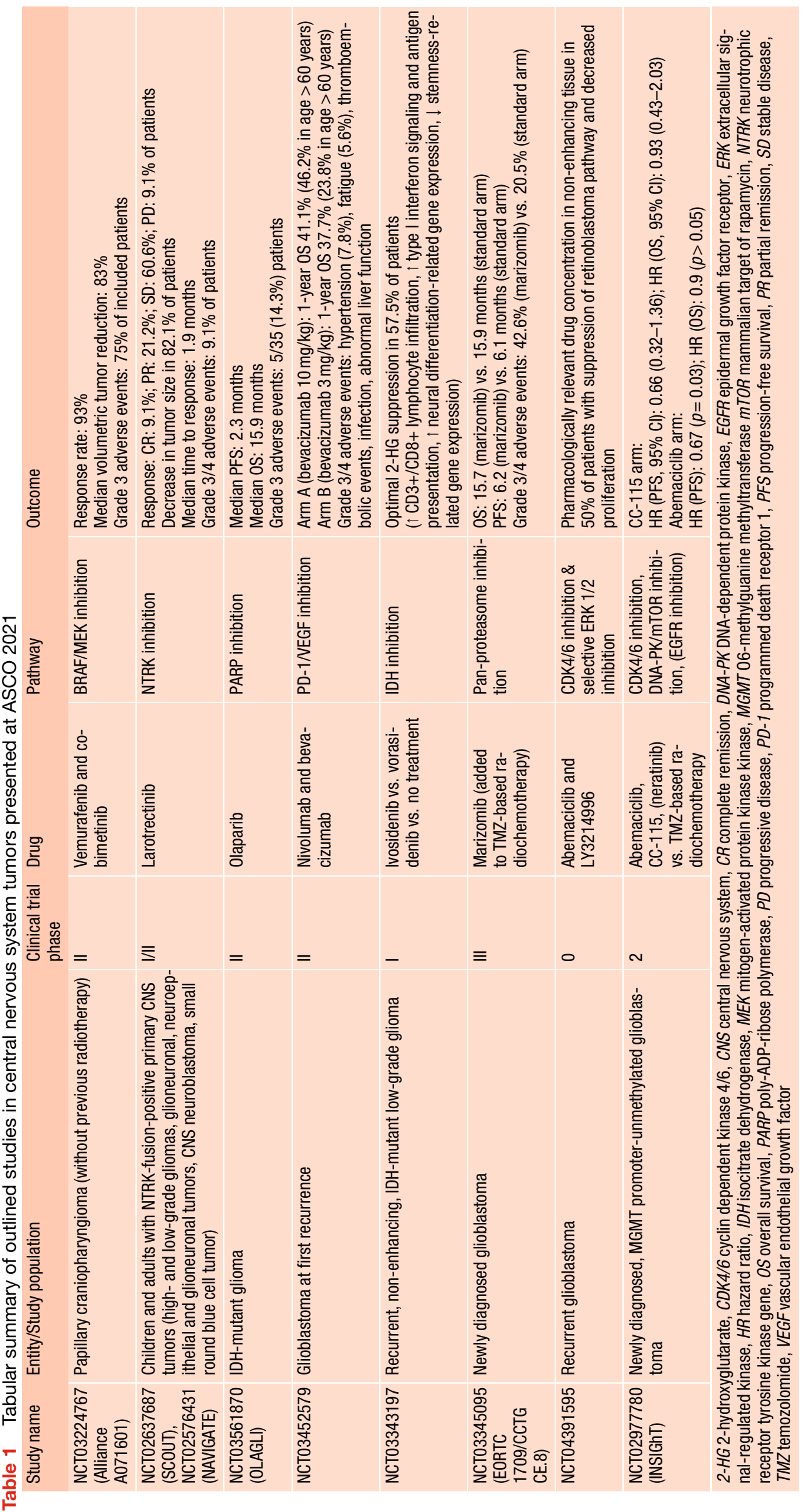


diochemotherapy [16]. A total of 749 patients was enrolled as the independent data monitoring committee recommended premature closure of the trial due to futility. Median OS was comparable between the two groups (15.7 months in the marizomib arm vs. 15.9 months in the standard arm). Similarly, no improvement in PFS was observed (6.2 months in the marizomib arm vs. 6.1 months in the standard arm). However, grade 3/4 adverse events were more frequent in the marizomib arm (42.6\% vs. $20.5 \%)$ and included those seen in previous trials (hallucinations, headache, ataxia). Further subgroup analyses are ongoing.

\section{"Thinking outside the box": novel clinical trial designs in neuro-oncology}

The EORTC-1709 study fits well into many negative phase III trials in GBM in the past decade. Phase III trials in newly diagnosed tumors are often based on an extrapolation of phase II data in recurrent disease. Conversely, many compounds are initially tested in recurrent, heavily pretreated GBM and do not reach evaluation in newly diagnosed disease. Further challenges of clinical trials in neuro-oncology include the biological heterogeneity of CNS tumors, primary resistance mechanisms and the role of the blood-brain barrier where translational research will provide further insights.

At ASCO 2021, some presentations proposed interesting trial designs which may help to overcome these hurdles. Data from a phase $0 / 2$ "trigger" trial of combined CDK4/6+ERK1/2 inhibition in recurrent GBM were shown [17]. Patients were required to have planned re-resection of recurrent GBM after radiochemotherapy with a contrast-enhancing tumor volume of at least $1 \mathrm{~cm}^{3}$. Furthermore, retinoblastoma $(\mathrm{RB})$ protein expression as well as either loss of the endogenous CDK inhibitors CDKN2A/B/C or an amplification of CDK4/6 and expression of pERK were mandatory, whereas RB gene mutations were not allowed. In the phase 0 part of the study, patients preoperatively received the CDK4/6 inhibitor abemaciclib and the ERK1/2 inhibitor LY3214996 for 6 days. Resection was performed 7-9h (arm A) or 3-5 h (arm B) after the last dose, and samples of the tumor, blood, and craniospinal fluid were obtained. A five-fold concentration of the IC50 (pharmacokinetic "trigger") could be detected in the nonenhancing tumor of 5/10 patients, who then underwent postoperative treatment with the study drugs (phase 2). In conclusion, pharmacologically relevant concentrations could be measured in the included tumors. Still, it remains to be awaited whether drug bioavailability in tumor tissue is accompanied by clinical efficacy.

Another promising trial design was applied in the Individualized Screening Trail of Innovative Glioblastoma Therapy (INSIGhT). This "adaptive platform" trial consisted of one control arm (TMZ-based radiochemotherapy) and three experimental arms with radiotherapy and (A) the $\mathrm{CDK} 4 / 6$ inhibitor abemaciclib, (B) DNA-PK/mTOR inhibitor CC-115 and (C) TMZ followed by the epidermal growth factor receptor inhibitor neratinib. The adaptive randomization approach allows to randomize patients based on early results of the ongoing trial. Thereby, randomization into arms with less active treatments is gradually decreased over time, while subjects are more likely to be enrolled in effective treatments. Similarly, randomization can be adjusted based on early results on predictive biomarkers. At ASCO 2021, the results of the CC-115 arm were presented [18]. In total, 12 patients were randomized to CC-115, and no significant benefit for progression-free and overall survival were seen. The probability of randomization to the CC-115 arm decreased from $25 \%$ to $16 \%$ due to early PFS data, and $50 \%$ less patients were randomized as compared to standard randomization, limiting the number of patients who received a less active treatment. In another presentation, the results of the abemaciclib arm were shown, with a significant increase in PFS, but no significant benefit in terms of OS while treatment was overall well tolerated [19]. Similar "platform" approaches are currently followed by the ongoing GBM AGILE [20] and the tumor-agnostic NCI-MATCH [21] trials.

\section{Take home message}

Practice-changing results were limited to very rare subgroups of CNS tumors such as those with NTRK fusions and BRAF V600E-positive craniopharyngioma. Although the EORTC-1709 phase III trial was overall negative, interesting approaches for future clinical trials were shown at ASCO 2021. Platform trials as well as innovative phase $0 / 2$ designs with a strong translational foundation have the potential to point towards innovative treatment modalities in neuro-oncology.

Funding Open access funding provided by Medical University of Vienna.

Conflict of interest M. J. Mair declares that he has no competing interests. A. S. Berghoff has received research support from Daiichi Sankyo, Roche, and honoraria for lectures, consultation or advisory board participation from Roche, BristolMeyers Squibb, Merck, Daiichi Sankyo as well as travel support from Roche, Amgen and AbbVie.

Open Access This article is licensed under a Creative Commons Attribution 4.0 International License, which permits use, sharing, adaptation, distribution and reproduction in any medium or format, as long as you give appropriate credit to the original author(s) and the source, provide a link to the Creative Commons licence, and indicate if changes were made. The images or other third party material in this article are included in the article's Creative Commons licence, unless indicated otherwise in a credit line to the material. If material is not included in the article's Creative Commons licence and 
your intended use is not permitted by statutory regulation or exceeds the permitted use, you will need to obtain permission directly from the copyright holder. To view a copy of this licence, visit http://creativecommons.org/licenses/by/4.0/.

\section{References}

1. Brastianos PK, Taylor-Weiner A, Manley PE, et al. Exome sequencing identifies BRAF mutations in papillary craniopharyngiomas. Nat Genet. 2014;46:161-5. https://doi.org/ 10.1038/ng.2868.

2. Brastianos PK, Twohy E, Geyer SM, et al. Alliance A071601: phase II trial of BRAF/MEK inhibition in newly diagnosed papillary craniopharyngiomas. J Clin Oncol. 2021;39:2000. https://doi.org/10.1200/JCO.2021.39.15_suppl.2000.

3. Hong DS, DuBois SG, Kummar S, et al. Larotrectinib in patients with TRK fusion-positive solid tumours: a pooled analysis of three phase $1 / 2$ clinical trials. Lancet Oncol. 2020;21:531-40. https://doi.org/10.1016/S14702045(19)30856-3.

4. Perreault S, van Tilburg CM, Geoerger B, et al. Efficacy and safety of larotrectinib in adult and pediatric patients with tropomyosin receptorkinase (TRK) fusion-positive primary central nervous system tumors. J Clin Oncol. 2021;39:2002. https://doi.org/10.1200/JCO.2021.39.15_suppl.2002.

5. Sulkowski PL, Corso CD, Robinson ND, et al. 2-Hydroxyglutarate produced by neomorphic IDH mutations suppresses homologous recombination and induces PARP inhibitor sensitivity. Sci Transl Med. 2017;9:eaal2463. https://doi. org/10.1126/scitranslmed.aal2463.

6. Ducray F, Sanson M, Chinot OL, et al. Olaparib in recurrent IDH-mutant high-grade glioma (OLAGLI). J Clin Oncol. 2021;39:2007. https://doi.org/10.1200/JCO.2021. 39.15_suppl.2007.

7. Mandel JJ, Cachia D, Liu D, et al. Impact of IDH1 mutation status on outcome in clinical trials for recurrent glioblastoma. J Neurooncol. 2016;129:147-54. https://doi.org/10. 1007/s11060-016-2157-2.

8. Reardon DA, Brandes AA, Omuro A, et al. Effect of nivolumab vs bevacizumab in patients with recurrent glioblastoma. JAMA Oncol. 2020;6:1003. https://doi. org/10.1001/jamaoncol.2020.1024.

9. Bristol Myers Squibb. Press release: Bristol-Myers Squibb announces phase 3 checkmate -498 study did not meet primary endpoint of overall survival with Opdivo (nivolumab) plus radiation in patients with newly diagnosed MGMTunmethylated glioblastoma multiforme. 2019. https:// news.bms.com/press-release/corporatefinancial-news/ bristol-myers-squibb-announces-phase-3-checkmate498-study-did. Accessed 13 Dec 2019.

10. Bristol Myers Squibb. Press release: Bristol-Myers Squibb provides update on phase 3 Opdivo (nivolumab) checkmate -548 trial in patients with newly diagnosed MGMTmethylated glioblastoma multiforme. 2019. https:// news.bms.com/press-release/corporatefinancial-news/ bristol-myers-squibb-provides-update-phase-3-opdivonivolumab-. Accessed 13 Dec 2019.

11. Fukumura D, Kloepper J, Amoozgar Z, et al. Enhancing cancer immunotherapy using antiangiogenics: opportunities and challenges. Nat Rev Clin Oncol. 2018;15:325-40. https://doi.org/10.1038/nrclinonc.2018.29.

12. Ahluwalia MS, RaufY, Li H, et al. Randomized phase 2 study of nivolumab (nivo) plus either standard or reduced dose bevacizumab (bev) in recurrent glioblastoma (rGBM). JClin Oncol. 2021;39:2015. https://doi.org/10.1200/JCO.2021. 39.15_suppl.2015.

13. Lu M, Cloughesy TF, Wen PY, et al. Impact of mutant IDH $(\mathrm{mIDH})$ inhibition on DNA hydroxymethylation, tumor cell function, and tumor immune microenvironment (TIME) in resected $\mathrm{mIDH} 1$ lower-grade glioma (LGG). J Clin Oncol. 2021;39:2008. https://doi.org/10.1200/JCO.2021. 39.15_suppl.2008.

14. Stupp R, Mason WP, van den Bent MJ, et al. Radiotherapy plus concomitant and adjuvant temozolomide for glioblastoma. N Engl J Med. 2005;352:987-96. https://doi.org/10. 1056/NEJMoa043330.

15. Mason WP, Kesari S, Stupp R, et al. Full enrollment results from an extended phase I, multicenter, open label study of marizomib (MRZ) with temozolomide (TMZ) and radiotherapy (RT) in newly diagnosed glioblastoma (GBM). J Clin Oncol. 2019;37:2021. https://doi.org/10.1200/JCO. 2019.37.15_suppl.2021.

16. Roth P, Gorlia T, Reijneveld JC, et al. EORTC 1709/CCTG CE.8: a phase III trial of marizomib in combination with temozolomide-based radiochemotherapy versus temozolomide-based radiochemotherapy alone in patients with newly diagnosed glioblastoma. J Clin Oncol. 2021;39:2004. https://doi.org/10.1200/JCO.2021.39.15_suppl.2004.

17. Sanai N, Chang Y-W, Margaryan T, et al. A phase 0 'trigger' trial of CDK4/ 6 plus ERK1/2 inhibitors in recurrent glioblastoma. J Clin Oncol. 2021;39:2005. https://doi.org/ 10.1200/JCO.2021.39.15_suppl.2005.

18. Rahman R, Trippa L, Fell G, et al. Evaluating the benefit of adaptive randomization in the CC-115 arm of the Individualized Screening Trial of Innovative Glioblastoma Therapy (INSIGhT): a phase II randomized Bayesian adaptive platform trial in newly diagnosed MGMTunmethylated glioblastoma. J Clin Oncol. 2021;39:2006. https://doi.org/ 10.1200/JCO.2021.39.15_suppl.2006.

19. Lee EQ, Trippa L, Fell G, et al. Preliminary results of the abemaciclib arm in the Individualized Screening Trial of Innovative Glioblastoma Therapy (INSIGhT): a phase II platform trial using Bayesian adaptive randomization. J Clin Oncol. 2021;39:2014. https://doi.org/10.1200/JCO. 2021.39.15_suppl.2014.

20. Buxton MB, Alexander BM, Berry DA, et al. GBM AGILE: a global, phase II/III adaptive platform trial to evaluate multiple regimens in newly diagnosed and recurrent glioblastoma. J Clin Oncol. 2020;38:TPS2579. https://doi. org/10.1200/JCO.2020.38.15_suppl.TPS2579.

21. Flaherty KT, Gray R, Chen A, et al. The molecular analysis for therapy choice (NCI-MATCH) trial: lessons for genomic trial design. J Natl Cancer Inst. 2020;112:1021-9. https:// doi.org/10.1093/jnci/djz245.

Publisher's Note Springer Nature remains neutral with regard to jurisdictional claims in published maps and institutional affiliations.

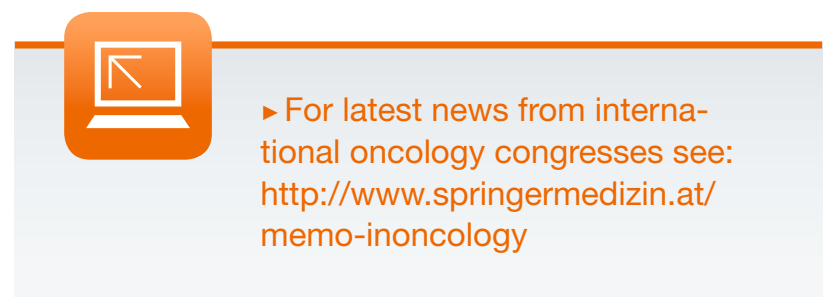

